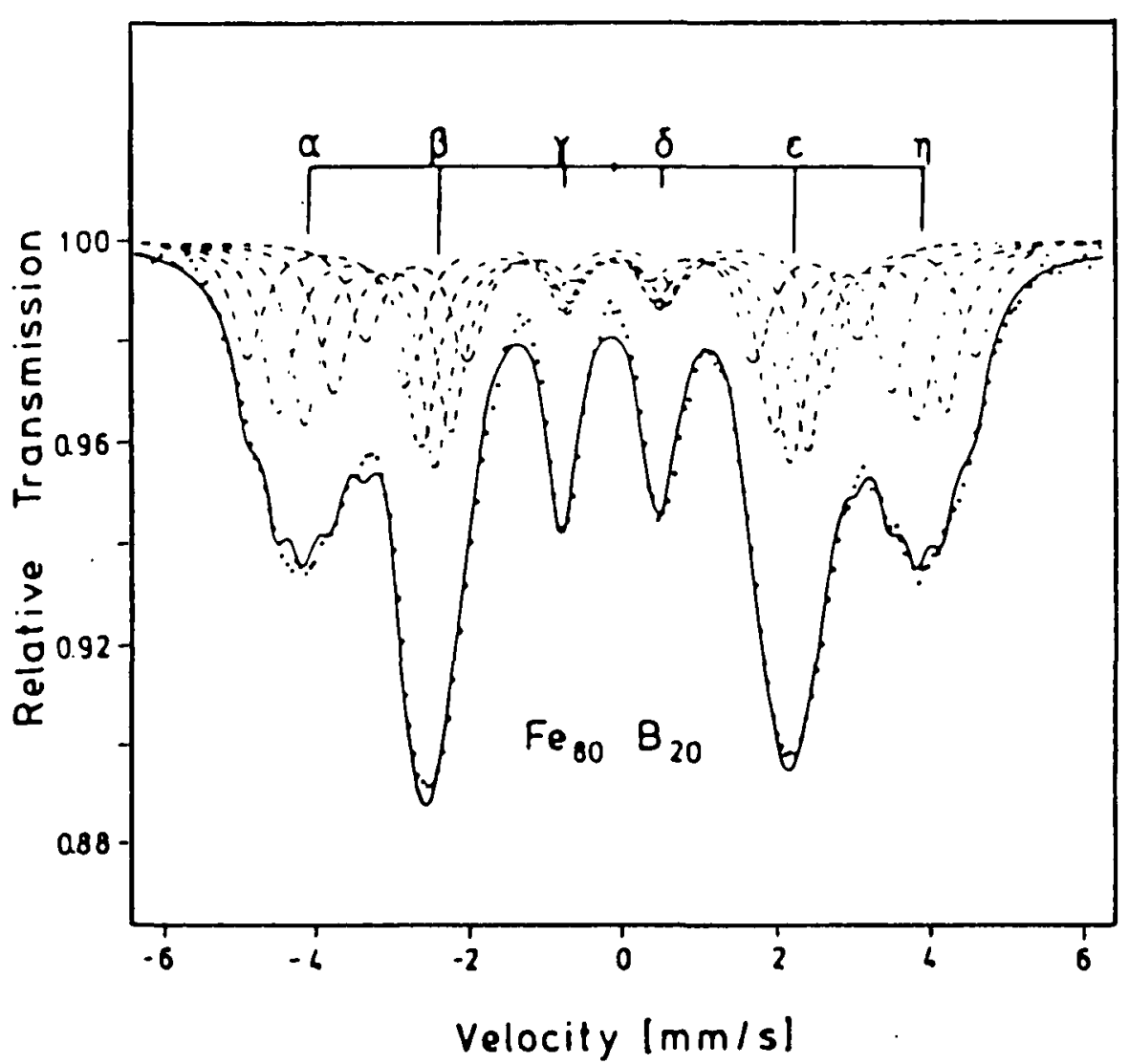

Figure 6. Mössbauer spectrum of the amorphous metal $\mathrm{Fe}_{80} \mathrm{~B}_{20}$. The stick diagram $(\alpha, \beta, \sigma, \delta, \varepsilon, \eta)$ represents the average hyperfine field. The solid line represents the sum of the subspectra. ${ }^{10}$ its application to the shuly of antimony compounds and fired-clay materials. His additional interests are with problems of evaluation and dissemination of scientific data and information.

\section{References}

1. R.L. Mössbauer, Z. Phys, 151 (1958) p. 124.

2. B.S. Clausen, S. Morup, H. Topsoe, and

R. Candia, J. Phys. Colloq. 37, C6-249 (1976).

3. H. Topsoe, in Surface Properties and Catalysts by Non-Metals: Oxides, Sulfilles, and Other Transition Metal Compounds, edited by J.P. Bonnelle, B. Delmon, and E. Derouane (Reidel, Dordrecht, 1983) p. 329.

4. C. Wivel, R. Candia, B.S. Clausen, S. Morup, and H. Topsoe, J. Catal. 68 (1981) p. 453.

5. H. Pollak, M. DeCoster, and S. Amelinckx, in Mösshaur Effert, edited by D.M.J. Compton and D. Sehuen (Wiley, New York, 1962) p. 298.

6. M.D. Dyar, American Mineralogist, 70 (1985) p. 304.

7. C.L. Chien and T. Hasegawa, J. Phys Colloq. 37, C6-759 (1976).

8. C.L. Chien, D. Musser, E.M. Gregory, R.C. Sherwood, H.S. Chen, F.E. Luborsky, and J.L. Walter, Phys. Re'v. B20 (1979) p. 283. 9. U. Gonser and R. Preston, in Glassy Metals 11 , edited by $\mathrm{H}$. Beck and J.-J. Guntherodt (Springer-Verlag, West Germany 1983) p. 93.

10. U. Gonser, in Atomic Energy Revirw, Supplement No. 1 (International Atomic Energy Agency, Vienna, 1981) p. 203.

\title{
MRS Book Publishing Program Expands
}

\author{
New Conference Proceedings Series Established, New Titles Sought
}

The Materials Research Society has recently expanded its book publishing program to include proceedings and other volumes spanning important materials research from sources other than MRS meetings. "A great deal of valuable scientific work is underway that the MRS publishing program wants to make available to MRS members. The new MRS Conference Proceedings series has been established to do just that in a timely way, according to MRS Publications Committee Chairman D.R. Campbell. The first volume in the new series, Tungsten and Other Refractory Metals for VLSI Applications, edited by R.S. Blewer, was published in 1986. To follow in early 1987 is the proceedings of the most recent workshop on this subject. Also scheduled for publication is the proceedings from the 7 th International Conference on Ternary and Multinary Compounds, held in late 1986. According to Campbell, approximately four additional volumes in the new series are also anticipated during the next year.

The series maintains the high standards set for the Society's own Symposia Proceedings series, explained P.P. Pronko, Chairman of the MRS Subcommittee on Books. Each book is reviewed by the Publications Committee for relevance to the Society, and selected titles are peer reviewed and published within 4-6 months following the meeting. "Tungsten and Other Refractory Mitals for VLSI Applications, which includes the proceedings of both the 1984 and 1985 workshops, has been extremely well received, indicating to us that this program is fulfilling a specific need in the profession," Pronko said. The MRS Symposia Proceedings series, which documents reports presented at the two annual MRS Meetings, now numbers 73 volumes, with a nother 15 volumes anticipated in 1987 . In addition to this series and the new Conference Proceedings series, the publishing program offers Extended Abstracts and the Society's two periodicals, Journal of Matirials Resenrch and MRS BULLETIN.

"Just as lourmal of Materials Resench will continue to expand in 1987 to include fulllength, original research articles and the BULLETIN to report news and trends affecting materials research, the book publishing program is anticipated to grow to encompass topics and formats to properly serve its readership," Campbell said. The Publications Committee welcomes inquiries from groups or individuals who wish to publish proceedings, monographs, textbooks, and other books on leadingedge materials research topics. Individuals interested in learning more about the MRS book publishing program, and how they might contribute, are encouraged to contact Dave Campbell at (914) 894-3075, Peter Pronko at (513) 426-6900, or Cuail Oare (MRS headquarters), (412) 367-3036. 


\section{MRS PUBLICATIONS}

\section{MRS MEETINGS 1980-83}

\section{Volume 1}

Laser and Electron-Beam Solid Interactions and Materials Processing

Editors: J.F. Gibbons, L.D. Hess, T.W. Sigmon, 1981

MRS Members- $\$ 36.00$

U.S. Nonmembers $-\$ 45.00$ Foreign Nonmembers $-\$ 55.00$

ISBN 0-444-00595-1

\section{Volume 6}

Scientific Basis for Nuclear Waste Management IV

Editor: S.V. Topp, 1982

MRS Members $-\$ 48.00$

U.S. Nonmembers- $\$ 60.00$ Foreign Nonmembers $-\$ 72.00$

ISBN 0-444-00699-0

\section{Volume 11}

Scientific Basis for Nuclear Waste Management V

Editor: W. Lutze, 1982

MRS Members- $\$ 48.00$

U.S. Nonmembers- $\$ 60.00$ Foreign Nonmembers $-\$ 72.00$

ISBN 0-444-00725-3

In-Situ Composites IV

Volume 12

Editors: F.D. Lemkey, H.E. Cline, M. McLean, 1982

MRS Members- $\$ 32.00$

U.S. Nonmembers $-\$ 40.00$ Foreign Nonmembers- $\$ 48.00$

ISBN 0-444-00726-1

\section{Volume 15}

Scientific Basis for Nuclear Waste Management VI

Editor: D.G. Brookins, 1983

MRS Members- $\$ 48.00$

U.S. Nonmembers $-\$ 60.00$ Foreign Nonmembers $-\$ 72.00$

ISBN 0-444-00780-6

\section{Volume 16}

Nuclear Radiation Detector Materials

Editors: E.E. Haller, H.W. Kraner, W.A. Higinbotham, 1983

MRS Members - $\$ 32.00$

U.S. Nonmembers $-\$ 40.00$ Foreign Nonmembers $-\$ 48.00$ ISBN 0-444-00787-3

Intercalated Graphite

Volume 20

Editors: M.S. Dresselhaus, G. Dresselhaus, J.E. Fischer, M.J. Moran, 1983

MRS Members- $\$ 36.00$

U.S. Nonmembers- $\$ 45.00$ Foreign Nonmembers $-\$ 55.00$ ISBN 0-444-00781-4

\section{Volume 26}

Scientific Basis for Nuclear Waste Management VII

Editor: G.L. McVay, 1984

MRS Members- $\$ 60.00$

U.S. Nonmembers $-\$ 72.00$ Foreign Nonmembers $-\$ 85.00$

ISBN 0-444-00906-X

\section{Volume 29}

Laser-Controlled Chemical Processing of Surfaces

Editors: A.W. Johnson, D.J. Ehrlich, H.R. Schlossberg, 1984 MRS Members- $\$ 40.00$

U.S. Nonmembers- $\$ 48.00$ Foreign Nonmembers $-\$ 60.00$

ISBN 0-444-00894-2

\section{Volume 30}

Plasma Processing and Synthesis of Materials

Editors: J. Szekeley, D. Apelian, 1984

MRS Members - $\$ 36.00$

U.S. Nonmembers- $\$ 45.00$ Foreign Nonmembers $-\$ 55.00$ ISBN 0-444-00895-0

\section{Volume 31}

Electron Microscopy of Materials

Editors: W. Krakow, D.A. Smith, L.W. Hobbs, 1984

MRS Members- $\$ 36.00$

U.S. Nonmembers $-\$ 45.00$ Foreign Nonmembers $-\$ 55.00$ ISBN 0-444-00897-7

Volume 32

Better Ceramics Through Chemistry

Editors: C.J. Brinker, D. Clark, D.R. Ulrich, 1984

MRS Members- $\$ 45.00$

U.S. Nonmembers $-\$ 55.00$ Foreign Nonmembers- $\$ 65.00$ ISBN 0-444-00898-5

\section{Volume 33}

Comparison of Thin Film Transistor and SOI Technologies Editors: H.W. Lam, M.J. Thompson, 1984

MRS Members $-\$ 45.00$

U.S. Nonmembers $-\$ 55.00$ Foreign Nonmembers $-\$ 65.00$ ISBN 0-444-00899-3

\section{Volume 34}

The Physical Metallurgy of Cast Iron

Editors: H. Fredriksson, M. Hillerts, 1985

MRS Members- $\$ 48.00$

U.S. Nonmembers - $\$ 53.00$ Foreign Nonmembers $-\$ 58.00$ ISBN 0-444-00938-8

\section{PROCEEDINGS FROM 1984 MRS FALL MEETING}

ISSN: 0272-9172

\section{Volume 35}

Energy Beam-Solid Interactions and Transient Thermal Processing/ 1984

Editors: D.K. Biegelsen, G.A. Rozgonyi, C.V. Shank, 1985 MRS Members- $\$ 36.00$

U.S. Nonmembers $-\$ 50.00$ Foreign Nonmembers $-\$ 55.00$ ISBN 0-931837-00-6

Volume 36

Impurity Diffusion and Gettering in Silicon

Editors: R.B. Fair, C.W. Pearce, J. Washburn, 1985

MRS Members- $\$ 25.00$

U.S. Nonmembers $-\$ 36.00$ Foreign Nonmembers $-\$ 43.00$ ISBN 0-931837-01-4

Volume 37

Layered Structures, Epitaxy, and Interfaces

Editors: J.M. Gibson, L.R. Dawson, 1985

MRS Members $-\$ 36.00$

U.S. Nonmembers- $\$ 50.00$ Foreign Nonmembers $-\$ 55.00$ ISBN 0-931837-02-2 
Volume 38

Plasma Synthesis and Etching of Electronic Materials Editors: R.P.H. Chang, B. Abeles, 1985

MRS Members- $\$ 30.00$

U.S. Nonmembers- $\$ 43.00$ Foreign Nonmembers- $\$ 52.00$ ISBN 0-931837-03-0

\section{Volume 39}

High-Temperature Ordered Intermetallic Alloys

Editors: C.C. Koch, C.T. Liu, N.S. Stoloff, 1985

MRS Members- $\$ 30.00$

U.S. Nonmembers $-\$ 43.00$ Foreign Nonmembers $-\$ 52.00$ ISBN 0-931837-04-9

\section{Volume 40}

Electronic Packaging Materials Science

Editors: E.A. Giess, K.-N. Tu, D.R. UhImann, 1985

MRS Members- $\$ 25.00$

U.S. Nonmembers- $\$ 36.00$ Foreign Nonmembers $-\$ 43.00$ ISBN 0-931837-05-7

Volume 41

Advanced Photon and Particle Techniques for the Characterization of Defects in Solids

Editors: J.B. Roberto, R.W. Carpenter, M.C. Wittels, 1985

MRS Members- $\$ 30.00$

U.S. Nonmembers $-\$ 43.00$ Foreign Nonmembers $-\$ 52.00$ ISBN 0-931837-06-5

\section{Volume 42}

Very High Strength Cement-Based Materials

Editor: J.F. Young, 1985

MRS Members- $\$ 25.00$

U.S. Nonmembers $-\$ 36.00$ Foreign Nonmembers $-\$ 43.00$ ISBN 0-931837-07-3

\section{Volume 43}

Fly Ash and Coal Conversion By-Products: Characterization, Utilization and Disposal 1

Editors: G.J. McCarthy, R.J. Lauf, 1985

MRS Members $-\$ 20.00$

U.S. Nonmembers $-\$ 30.00$ Foreign Nonmembers- $\$ 36.00$ ISBN 0-931837-08-1

Volume 44

Scientific Basis for Nuclear Waste Management VIII

Editors: C.M. Jantzen, J.A. Stone, R.C. Ewing, 1985

MRS Members- $\$ 45.00$

U.S. Nonmembers- $\$ 55.00$ Foreign Nonmembers- $\$ 60.00$

ISBN 0-931837-09-X

\section{PROCEEDINGS FROM 1985 MRS SPRING MEETING}

ISSN: $0272-9172$

\section{Volume 45}

Ion Beam Processes in Advanced Electronic Materials and Device Technology

Editors: B.A. Appleton, F.H. Eisen, T.W. Sigmon, 1985 MRS Members- $\$ 35.00$

U.S. Nonmembers $-\$ 42.00$ Foreign Nonmembers $-\$ 48.00$ ISBN 0-931837-10-3

\section{Volume 46}

Microscopic Identification of Electronic Defects in Semiconductors

Editors: N.M. Johnson, S.G. Bishop, G.D. Watkins, 1985 MRS Members- $\$ 41.00$

U.S. Nonmembers $-\$ 50.00$ Foreign Nonmembers $-\$ 57.00$ ISBN 0-931837-11-1
Volume 47

Thin Films: The Relationship of Structure to Properties Editors: C.R. Aita, K.S. SreeHarsha, 1985

MRS Members- $\$ 30.00$

U.S. Nonmembers $-\$ 35.00$ Foreign Nonmembers $-\$ 42.00$ ISBN 0-931837-12-X

Volume 48

Applied Materials Characterization

Editors: W. Katz, P. Williams, 1985

MRS Members- $\$ 41.00$

U.S. Nonmembers $-\$ 50.00$ Foreign Nonmembers $-\$ 57.00$ ISBN 0-931837-13-8

\section{Volume 49}

Materials Issues in Applications of Amorphous Silicon Technology

Editors: D. Adler, A. Madan, M.J. Thompson, 1985

MRS Members- $\$ 35.00$

U.S. Nonmembers $-\$ 42.00$ Foreign Nonmembers $-\$ 48.00$ ISBN 0-931837-14-6

\section{PROCEEDINGS FROM NINTH SYMPOSIUM ON NUCLEAR WASTE MANAGEMENT}

Volume 50

Scientific Basis for Nuclear Waste Management IX

Editor: L.O. Werme, 1986

MRS Members - $\$ 50.00$

U.S. Nonmembers $-\$ 55.00$ Foreign Nonmembers $-\$ 60.00$ ISBN 0-931837-15-4

\section{PROCEEDINGS FROM 1985 MRS FALL MEETING}

ISSN: 0272-9172

\section{Volume 51}

Beam-Solid Interactions and Phase Transformations Editors: H. Kurz, G.L. O/son, J.M. Poate, 1986 MRS Members- $\$ 37.00$

U.S. Nonmembers- $\$ 46.00$ Foreign Nonmembers $-\$ 51.00$ ISBN 0-931837-16-2

Rapid Thermal Processing

Volume 52

Editors: T.O. Sedgwick, T.E. Seidel, B.-Y. Tsaur, 1986 MRS Members- $\$ 35.00$

U.S. Nonmembers $-\$ 44.00$ Foreign Nonmembers $-\$ 49.00$ ISBN 0-931837-17-0

\section{Volume 53}

Semiconductor-on-Insulator and Thin Film Transistor Technology

Editors: A. Chiang, M.W. Geis, L. Pfeiffer, 1986

MRS Members - $\$ 37.00$

U.S. Nonmembers $-\$ 46.00$ Foreign Nonmembers- $\$ 51.00$ ISBN 0-931837-18-9

\section{Volume 54}

Thin Films-Interfaces and Phenomena Editors: R.J. Nemanich, P.S. Ho, S.S. Lau, 1986 MRS Members- $\$ 45.00$

U.S. Nonmembers- $\$ 55.00$ Foreign Nonmembers $-\$ 59.00$ ISBN 0-931837-19-7 


\section{Volume 55}

Biomedical Materials

Editors: J.M. Williams, M.F. Nichols, W. Zingg, 1986

MRS Members- $\$ 37.00$

U.S. Nonmembers- $\$ 46.00$ Foreign Nonmembers $-\$ 51.00$ ISBN 0-931837-20-0

\section{Volume 56}

Layered Structures and Epitaxy

Editors: J.M. Gibson, G.C. Osbourn, R.M. Tromp, 1986

MRS Members- $\$ 39.00$

U.S. Nonmembers $-\$ 49.00$ Foreign Nonmembers $-\$ 54.00$ ISBN 0-931837-21-9

\section{Volume 57}

Phase Transitions in Condensed Systems-Experiments and Theory

Editors: G.S. Cargill III, F. Spaepen, K.-N. Tu, 1987

MRS Members - $\$ 37.00$

U.S. Nonmembers $-\$ 46.00$ Foreign Nonmembers $-\$ 51.00$ ISBN 0-931837-22-7

\section{Volume 58}

Rapidly Solidified Alloys and Their Mechanical and Magnetic Properties

Editors: B.C. Giessen, D.E. Polk, A.I. Taub, 1986

MRS Members- $\$ 33.00$

U.S. Nonmembers $-\$ 41.00$ Foreign Nonmembers $-\$ 46.00$ ISBN 0-931837-23-5

\section{Volume 59}

Oxygen, Carbon, Hydrogen, and Nitrogen in Crystalline Silicon

Editors: J.C. Mikkelsen, Jr., S.J. Pearton, J.W. Corbett, S.J. Pennycook, 1986

MRS Members - $\$ 37.00$

U.S. Nonmembers $-\$ 46.00$ Foreign Nonmembers $-\$ 51.00$ ISBN 0-931837-24-3

\section{Volume 60}

Defect Properties and Processing of High-Technology Nonmetallic Materials

Editors: Y. Chen, W.D. Kingery, R.J. Stokes, 1986

MRS Members- $\$ 38.00$

U.S. Nonmembers $-\$ 48.00$ Foreign Nonmembers $-\$ 53.00$ ISBN 0-931837-25-1

\section{Volume 61}

Defects in Glasses

Editors: F.L. Galeener, D.L. Griscom, M.J. Weber, 1986

MRS Members - $\$ 33.00$

U.S. Nonmembers $-\$ 41.00$ Foreign Nonmembers $-\$ 46.00$ ISBN 0-931837-26-X

\section{Volume 62}

Materials Problem Solving with the Transmission Electron Microscope

Editors: L.W. Hobbs, K.H. Westmacott, D.B. Williams, 1986 MRS Members- $\$ 37.00$

U.S. Nonmembers $-\$ 46.00$ Foreign Nonmembers $-\$ 51.00$ ISBN 0-931837-27-8

\section{Volume 63}

Computer-Based Microscopic Description of the Structure and Properties of Materials

Editors: J. Broughton, W. Krakow, S.T. Pantelides, 1986 MRS Members - $\$ 35.00$

U.S. Nonmembers $-\$ 44.00$ Foreign Nonmembers $-\$ 49.00$ ISBN 0-931837-28-6

\section{Volume 64}

Cement-Based Composites: Strain Rate Effects on Fracture Editors: S. Mindess, S.P. Shah, 1986

MRS Members- $\$ 29.00$

U.S. Nonmembers $-\$ 36.00$ Foreign Nonmembers $-\$ 43.00$ ISBN 0-931837-29-4
Volume 65

Fly Ash and Coal Conversion By-Products: Characterization, Utilization and Disposal II

Editors: G.J. McCarthy, F.P. Glasser, D.M. Roy, 1986

MRS Members- $\$ 29.00$

U.S. Nonmembers $-\$ 36.00$ Foreign Nonmembers $-\$ 43.00$ ISBN 0-931837-30-8

Volume 66

Frontiers in Materials Education

Editors: L.W. Hobbs, G.L. Liedl, 1986

MRS Members- $\$ 29.00$

U.S. Nonmembers $-\$ 36.00$ Foreign Nonmembers $-\$ 43.00$ ISBN 0-931837-31-6

\section{PROCEEDINGS FROM 1986 MRS SPRING MEETING}

\section{ISSN 0272-9172}

Heteroepitaxy on Silicon

\author{
Volume 67
}

Editors: J.C.C. Fan, J.M. Poate, 1986

MRS Members- $\$ 34.00$

U.S. Nonmembers- $\$ 40.00$ Foreign Nonmembers $-\$ 44.00$ ISBN 0-931837-33-2

Plasma Processing

Volume 68

Editors: J.W. Coburn, R.A. Gottscho, D.W. Hess, 1986 MRS Members $-\$ 37.00$

U.S. Nonmembers- $\$ 44.00$ Foreign Nonmembers $-\$ 49.00$ ISBN 0-931837-34-0

Materials Characterization

\section{Volume 69}

Editors: N.W. Cheung, M.-A. Nicolet, 1986

MRS Members- $\$ 37.00$

U.S. Nonmembers $-\$ 44.00$ Foreign Nonmembers $-\$ 49.00$ ISBN 0-931837-35-9

\section{Volume 70}

Materials Issues in Amorphous-Semiconductor Technology Editors: D. Adler, Y. Hamakawa, A. Madan, 1986 MRS Members $-\$ 41.00$

U.S. Nonmembers $-\$ 48.00$ Foreign Nonmembers- $\$ 54.00$ ISBN 0-931837-36-7

\section{Volume 71}

Materials Issues in Silicon Integrated Circuit Processing Editors: M. Wittmer, J. Stimmell, M. Strathman, 1986 MRS Members $-\$ 37.00$

U.S. Nonmembers $-\$ 44.00$ Foreign Nonmembers $-\$ 49.00$ ISBN 0-931837-37-5

\section{Volume 72}

Electronic Packaging Materials Science II

Editors: K.A. Jackson, R.C. Pohanka, D.R. Uhimann, D.R. Ulrich, 1986

MRS Members- $\$ 33.00$

U.S. Nonmembers $-\$ 38.00$ Foreign Nonmembers $-\$ 44.00$ ISBN 0-931837-38-3

\section{Volume 73}

Better Ceramics Through Chemistry II

Editors: C.J. Brinker, D.E. Clark, D.R. Ulrich, 1986 MRS Members- $\$ 35.00$

U.S. Nonmembers $-\$ 41.00$ Foreign Nonmembers $-\$ 46.00$ ISBN 0-931837-39-1 


\section{MRS FALL MEETING}

The following prices are effective until March 30, 1987. Prices after this date will be higher.

Order your copies today to receive priority shipment.

ISSN: 0272-9172 Hardbound, indexed, illustrated.

\section{Volume 74 Symposium A}

Beam-Solid Interactions and Transient Processes

Editors: S. T. Picraux, M.O. Thompson, J.S. Williams, 1987 MRS Members $\$ 34.00$

U.S. Nonmembers $\$ 39.00 \quad$ Foreign Nonmembers $\$ 44.00$

ISBN 0-931837-40-5

Volume 75 Symposium $B$

Photon, Beam and Plasma Stimulated Chemical Processes at Surfaces

Editors: V.M. Donnelly, I.P. Herman, M. Hirose, 1987

MRS Members $\$ 34.00$

U.S. Nonmembers $\$ 39.00 \quad$ Foreign Nonmembers $\$ 44.00$

ISBN 0-931837-4†-3

\section{Volume 76 Symposium C}

Science and Technology of Microfabrication

Editors: R.E. Howard, E.L. Hu, S. Pang, S. Namba, 1987

MRS Members $\$ 32.00$

U.S. Nonmembers $\$ 38.00 \quad$ Foreign Nonmembers $\$ 43.00$

ISBN 0-931837-42-1

Volume 77 Symposium D

Interfaces, Superlattices, and Thin Films

Editors: J.D. Dow, I.K. Schuller, 1987

MRS Members $\$ 42.00$

U.S. Nonmembers $\$ 48.00 \quad$ Foreign Nonmembers $\$ 52.00$

ISBN 0-931837-56-1

Volume 78 Symposium E

Advances in Structural Ceramics

Editors: P.F. Becher, M.V. Swain, S. Somiya, 1987

MRS Members $\$ 26.00$

U.S. Nonmembers $\$ 32.00 \quad$ Foreign Nonmembers $\$ 37.00$

ISBN 0-931837-43-X

$$
\text { Volume } 79 \text { Symposium } F
$$

Scattering, Deformation and Fracture in Polymers

Editors: B. Crist, T.P. Russell, E.L. Thomas, G.D. Wignall, 1987

MRS Members $\$ 26.00$

U.S. Nonmembers $\$ 32.00$

ISBN 0-931837-44-8

\section{Volume 80 Symposium $\mathrm{G}$}

Science and Technology of Rapidly Quenched Alloys

Editors: M. Tenhover, L.E. Tanner, W.L. Johnson, 1987

MRS Members $\$ 34.00$

U.S. Nonmembers $\$ 39.00 \quad$ Foreign Nonmembers $\$ 44.00$

ISBN 0-931837-45-6

\section{Volume 81 Symposium $H$}

High-Temperature Ordered Intermetallic Alloys, II

Editors: C.C. Koch, C.T. Liu, N.S. Stoloff, O. Izumi, 1987

MRS Members $\$ 30.00$

U.S. Nonmembers $\$ 38.00 \quad$ Foreign Nonmembers $\$ 48.00$ ISBN 0-931837-46-4

$$
\text { Volume } 82 \text { Symposium I }
$$

Characterization of Defects in Materials

Editors: R.W. Siegel, R. Sinclair, J.R. Weertman, 1987

MRS Members $\$ 32.00$

U.S. Nonmembers $\$ 37.00 \quad$ Foreign Nonmembers $\$ 42.00$

ISBN 0-931837-47-2

Volume 83 Symposium $\mathrm{J}$

Physical and Chemical Properties of Thin Metal Overlayers and Alloy Surfaces

Editors: D.M. Zehner, D.W. Goodman, 1987

MRS Members $\$ 26.00$

U.S. Nonmembers $\$ 31.00 \quad$ Foreign Nonmembers $\$ 36.00$

ISBN 0-931837-48-0
Volume 84 Symposium L

Scientific Basis for Nuclear Waste Management, $X$

Editors: J.K. Bates, W.B. Seefe/dt, 1987

MRS Members $\$ 45.00$

U.S. Nonmembers $\$ 50.00 \quad$ Foreign Nonmembers $\$ 55.00$ ISBN 0-931837-49-9

Volume 85 Symposium $M$

Microstructural Development During the Hydration of Cement

Editors: L. Struble, P. Brown, 1987

MRS Members $\$ 29.00$

U.S. Nonmembers $\$ 34.00 \quad$ Foreign Nonmembers $\$ 39.00$ ISBN 0-931837-50-2

Volume 86 Symposium $N$

Fly Ash and Coal Conversion By-Products Characterization, Utilization and Disposal III

Editors: G.J. Mc Carthy, F.P. Glasser, D.M. Roy, S. Diamond, 1987

MRS Members $\$ 32.00$

U.S. Nonmembers $\$ 37.00 \quad$ Foreign Nonmembers $\$ 42.00$ ISBN 0-931837-51-0

\section{Volume 87 Symposium $O$}

Materials Processing in the Reduced Gravity Environment of Space

Editors: R.H. Doremus, P.C. Nordine, 1987

MRS Members $\$ 26.00$

U.S. Nonmembers $\$ 29.00 \quad$ Foreign Nonmembers $\$ 33.00$

ISBN 0-931837-52-9

$$
\text { Volume } 88 \text { Symposium P }
$$

Optical Fiber Materials and Properties

Editors: S.R. Nagel, G. Sigel, J.W. Fleming, D.A. Thompson, 1987

MRS Members $\$ 28.00$

U.S. Nonmembers $\$ 34.00 \quad$ Foreign Nonmembers $\$ 39.00$

ISBN 0-931837-53-7

Volume 89 Symposium $Q$

Diluted Magnetic (Semimagnetic) Semiconductors

Editors: J.K. Furdyna, R.L. Aggarwal, S. von Molnar, 1987

MRS Members $\$ 27.00$

U.S. Nonmembers $\$ 32.00 \quad$ Foreign Nonmembers $\$ 38.00$

ISBN 0-931837-54-5

$$
\text { Volume } 90 \quad \text { Symposium } R
$$

Materials for Infrared Detectors and Sources

Editors: R.F.C. Farrow, J.F. Schetzina, J.T. Cheung, 1987

MRS Members $\$ 27.00$

U.S. Nonmembers $\$ 32.00 \quad$ Foreign Nonmembers $\$ 38.00$

ISBN 0-931837-55-3 


\section{E-MRS EUROPE PROCEEDINGS}

\section{STRASBOURG - JUNE 5-8, 1984 \\ Code-R-1}

Poly-Micro-Crystalline and Amorphous Semiconductors

Editors: P. Pinard, S. Kalbitzer

MRS Members $\$ 52.00$

U.S. Nonmembers $\$ 62.00$

Code-R-2

Amorphous Metals and Non-Equilibrium Processing

Editor: $M$. Von Allmen

MRS Members $\$ 40.00$

U.S. Nonmembers $\$ 50.00$

Induced Defects in Insulators

Editor: P. Mazzoldi

MRS Members $\$ 34.00$

U.S. Nonmembers $\$ 44.00$

\section{STRASBOURG - MAY 13-15, 1985}

Code-R-4

Energy Beam Solid Interactions and Transient Thermal Processing Editors: V.T. Nguyen, A.G. Cullis

MRS Members $\$ 70.00$

U.S Nonmembers $\$ 80.00$

Code-R-5

Materials Under Extreme Conditions

Editors: H. Ahlborn, H. Fredriksson, E. Luscher

MRS Members $\$ 35.00$

U.S. Nonmembers $\$ 45.00$

Code-R-6

Semiconductor Quantum Well Structures and Superlattices

Editors: K. Ploog. N.T. Linh

MRS Members $\$ 42.00$

U.S. Nonmembers $\$ 52.00$

STRASBOURG - NOVEMBER 26-28, 1985

Code-R-7

Advanced Materials Research and Developments for Transport Light Metals

Editors: R.J.H. Wanhill, W.J.G. Bunk, J.G. Wurm

MRS Members $\$ 52.00$

U.S. Nonmembers $\$ 62.00$
Code-R-8

Advanced Materials Research and Developments for Transport Composites

Editors: P. Lamicq, W.J.G. Bunk, J.G. Wurm

MRS Members $\$ 52.00$

U.S. Nonmembers $\$ 62.00$

Code-R-9

Advanced Materials Research and Developments for Transport Ceramic Coatings for Heat Engines

Editors: I. Kvernes, W.J.G. Bunk, J.G. Wurm

MRS Members $\$ 52.00$

U.S. Nonmembers $\$ 62.00$

STRASBOURG - JUNE 17-20, 1986

Code R-10

Magnetic Thin Films

Editors: "

MRS Members $\$ 31.00$

U.S. Nonmembers $\$ 4100$

Code R-11

Laser Processing and Diagnostics

Editors: D. Bauerle, K.L. Kompa, L. Laude

MRS Members $\$ 49.00$

U.S. Nonmembers $\$ 59.00$

Code R-12

Dielectric Layers in Semiconductors-Novel Technologies and Devices

Editors: G. Bentini, E. Fogarassy, A. Golanski

MRS Members $\$ 56.00$

U.S. Nonmembers $\$ 66.00$

Code R-13

Advanced Materials for Telecommunications

Editors: P.A. Glasow, J.P. Noblanc, Y. Nissim, J Speight

MRS Members \$

U.S. Nonmembers \$ *

Code R-14

State of the Art of Computer Simulation of Casting and Solidification Processes

Editors: H. Frederiksson, W. Wurz, G. Lesoult, P. Sahm

MRS Members $\$$.

U.S. Nonmembers $\$$ *

- Information was not available at press time.

Outside the U.S., order from Les Editions de Physique, Avenue du Hoggar, B.P. 112, Zone Industrielle de Courtaboeuf, 91944 Les Ulis-Cedex, France; telephone 9073688 .

\section{CONFERENCE PROCEEDINGS/OTHER BOOKS}

\section{Code-VLSI Tungsten and Other Refractory Metals for VLSI Applications}

Editor: R.S. Blewer

This proceeding volume contains 59 papers that comprised both the 1984 and 1985 Workshops on Tungsten and Other Refractory Metals for VLSI Applications, sponsored by Sandia National Laboratories and the University of California-Berkeley's University Engineering Extension. Tungsten's self-aligning characteristics when applied by low-pressure chemical vapor deposition (LPCVD) and its high electrical conductivity make it ideal for use in advanced microcircuits and in source/drain covers. via fills and low-resistance shunts on polysilicon lines.

MRS Members $\$ 34.00$

U.S. Nonmembers $\$ 39.00$

Foreign Nonmembers $\$ 45.00$

Code-ARMS Vol. 16 Annual Review of Materials Science

Editors: R.A. Huggins, J.A. Giordmaine, J.B. Wachtman, Jr.

As a member of the Materials Research Society, you may purchase the 1986 volume of Annual Review of Materials Science at a special price- $\$ 10$ off the publisher's retail price. This annual publication assesses current trends in materials science from an interdisciplinary viewpoint. Emphasis is on general aspects of structure. characterization, phenomena, and properties related to materials engineering. This volume is an important summary of developments you need to know about. MRS Members $\$ 54.00$

Foreign Members $\$ 57.00$

\section{Advancing Materials Research}

From the National Research Council Published by National Academy Press

Highlights ancient and recent advances in materials science and engineering. provides a look at the current status of material research, and identifies major policy issues for corporate, academic and governmental services.

MRS Members $\$ 42.50$

U.S. Nonmembers $\$ 47.50$

Semiconductor-Based Heferostructures

Editors: M.L. Green, J.E.E. Baglin, G.Y. Chin, H. Deckman, W. Mayo, D. Narasinham

Proceedings of the 1986 TMS Northeast Regional Meeting, Cosponsored by Materials Research Society, contains essential information for materials scientists involved in electronic materials. This volume focuses on the structure and stablity of interfaces in a variety of semiconductor-based device structures.

MRS Members $\$ 50.00$

U.S. Nonmembers $\$ 73.00$

Pearson's Handbook of Crystallographic Data for Intermetallic Phases

By P. Villars and L.D. Calvert Published by American Society for Metals

MRS members may purchase the 1985 edition of this important handbook at special MRS member prices.

MRS Member Price/Set $\$ 370$

Regular Price/Set $\$ 420$ 


\section{MRS PUBLICATION ORDER FORM}

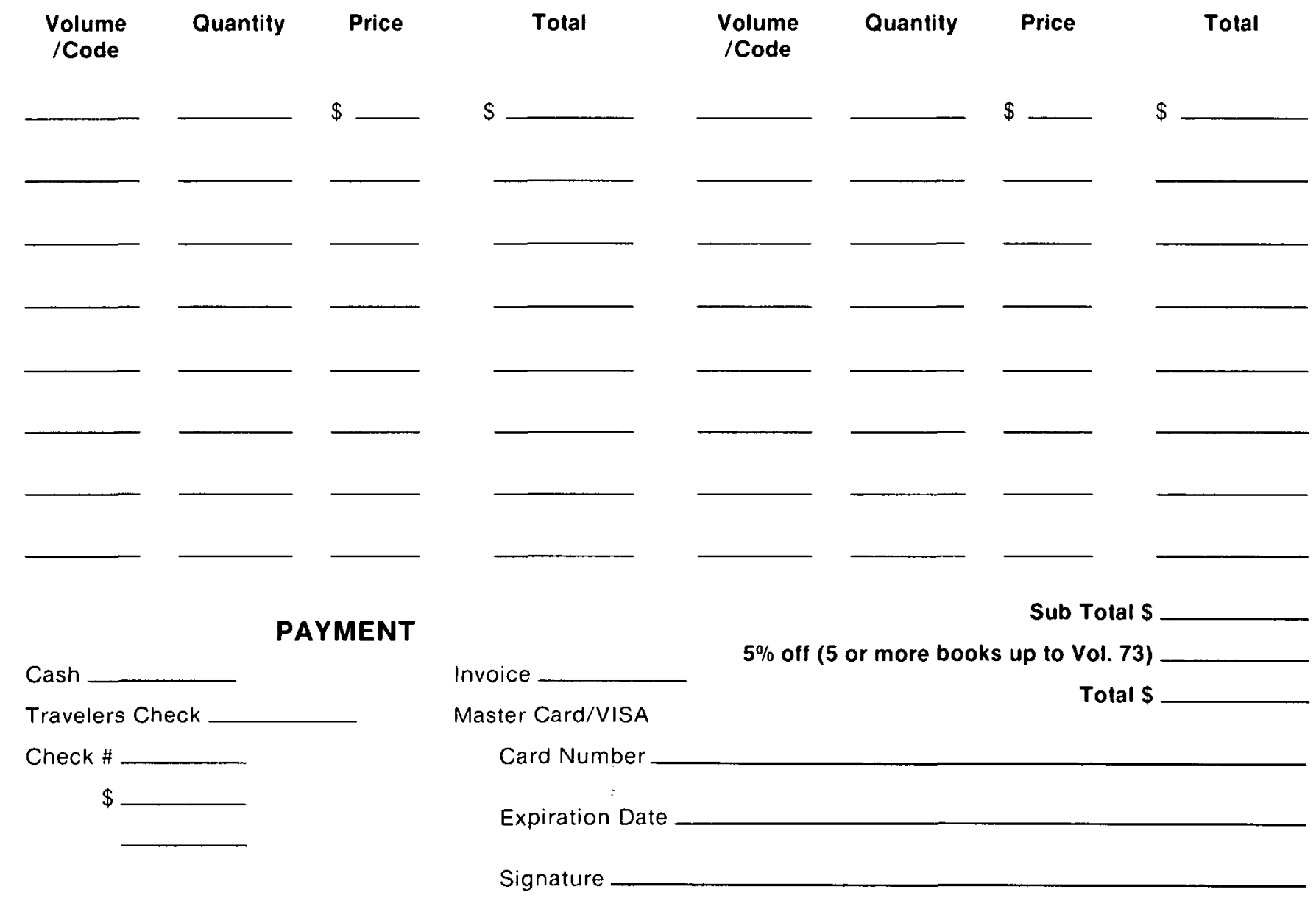

\section{Bill To}

Name

Organization

Address

City State

Zip County

Ship To

Name

Organization

Address

City State

Zip County 